Highly Energetic Physical Processes and

Mechanisms for Emission from Astrophysical Plasmas

IAU Symposium, Vol. 195, 2000

P. C. H. Martens, S. Tsuruta, and M. A. Weber, eds.

\title{
Internal Rotation of AB Doradus
}

\author{
K. M. Hiremath \\ Indian Institute of Astrophysics, Bangalore-560034, India
}

\begin{abstract}
Using Chandrasekhar's MHD equations, we solve for the steady part of the internal rotation of $\mathrm{AB}$ Doradus. We estimate the size of the convective envelope to be $\sim 40 \%$ of the radius and the rotation velocity at the base to be not less than $1.42 \times 10^{-4} \mathrm{rad} / \mathrm{sec}$. This study also yields the steady part of the toroidal magnetic field which is distributed throughout the convective envelope. We present rotational and toroidal magnetic field profiles in the interior and conjecture on the time-dependent part of the magnetic field.
\end{abstract}

\section{Introduction}

The K0 dwarf AB Doradus is a low-mass, rapidly rotating $(\sim 50 \times$ the equatorial rotation rate of the Sun) star which possesses a complex spot distribution over its surface. Recently, Donati \& Cameron (1997) found that the magnetic field of the star is time-dependent and that its surface rotation is differential. Assuming that the dominant part of the star's rotation is steady, we (Hiremath \& Gokhale 1996) recently solved Chandrasekhar's MHD equations (1956) for the internal rotation and for the toroidal part of the magnetic field. The solution yields isorotation contours similar to those inferred by helioseismology. Inspired by this study, we solve Chandrasekhar's MHD equations for the steady part of the internal rotation in the region of the convective envelope of $A B$ Doradus.

\section{Results and Discussion}

First, from the analytical solution of Chandrasekhar's MHD equations, we estimate the core rotation to be $\sim 1.42 \times 10^{-4} \mathrm{rad} \mathrm{sec}^{-1}$. Using the limit on the core rotation as a boundary condition at the base of the convection zone and the observed rotation as a boundary condition at the surface, we obtain a rotation profile in the convective envelope whose radial variation for the three latitude zones ( equator, $40.5^{\circ}$, and $71.8^{\circ}$ ) is presented in Figure 1a. It is interesting to note that the radial variation is similar to the radial variation of the Sun's rotation in the region of convective envelope.

For a consistent solution, and for a stellar radius of $5.95 \times 10^{10} \mathrm{~cm}$ (Allen 1972), we estimate the size of the convective envelope to be $40 \%$ of the radius. Correspondingly, the solution also yields the toroidal part of the magnetic field $(T)$ whose latitudinal variation for different radii is presented in Figure 1b. In this solution, $T$ is normalized to the mean density of $1.78 \mathrm{~g} \mathrm{~cm}^{-3}$ (Allen 1972). 

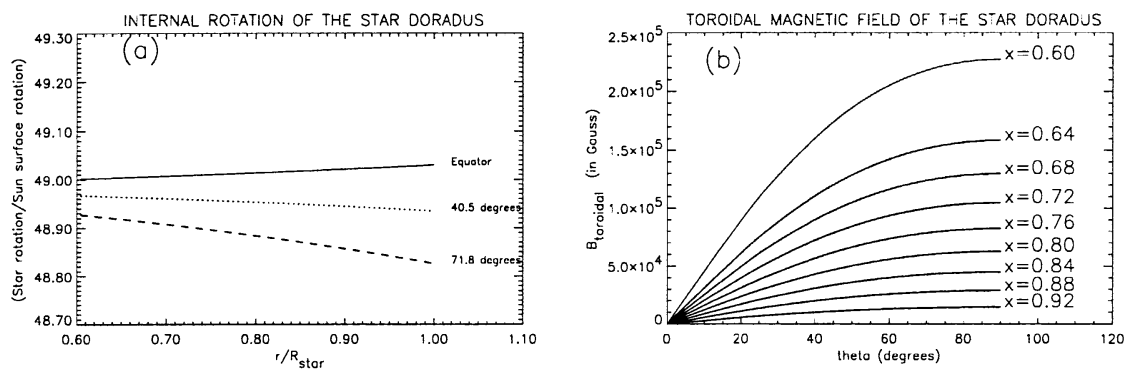

Figure 1. (a) The radial $\left(x=r / R_{\text {star }}\right)$ variation in rotation; and (b) the latitudinal variation of the magnetic field's toroidal component in the convective envelope of $\mathrm{AB}$ Doradus. The rotation is normalized to the Sun's surface equatorial rotation rate.

It may be noted that the field structure thus obtained is steady, and that we can not compare it with the observed magnetic field. However, we can make some conjectures on the time-dependent part of the toroidal magnetic field. If we follow the dynamo theories adopted to explain the time-dependent part of the magnetic field, the toroidal field is determined (Levy 1992) as follows. The toroidal field $\sim L \Omega B_{p} / \eta$, where $\mathrm{L}$ is the characteristic size of the convective envelope, $B_{p}$ is the strength of poloidal magnetic field, and $\eta$ is the magnetic eddy diffusivity. By taking typical values for $\mathrm{AB}$ Doradus as $L \sim 10^{10} \mathrm{~cm}$, $\Omega=1.4 \times 10^{-4} \mathrm{rad} / \mathrm{sec}\left(\sim 10^{6} \mathrm{~cm} / \mathrm{sec}\right)$, and $\eta \sim 10^{12} \mathrm{~cm}^{2} \mathrm{sec}^{-1}$, we get the strength of the toroidal field to be $\sim 10^{7} \mathrm{G}$. However, this result contradicts the observations of Donati \& Cameron (1997) which give the inferred toroidal field as $\sim 10^{3} \mathrm{G}$. This raises doubts about the applicability of so-called dynamo theories to this star. An alternative is that the toroidal field (which may be primordial in origin) is distributed throughout the convective envelope. The active regions are formed from the toroidal perturbations of the underlying field, leading to instabilities and bringing perturbed toroidal field to the surface. For example, if the resulting Alfvén-type perturbations have strengths similar to the strengths of the ambient steady magnetic field, then the observed fields of $800 \mathrm{G}$ might have formed just beneath the surface. However, the validity and details of such a mechanism remain to be worked out.

Acknowledgments. The author is grateful to the SOC and LOC of IAU Symposium 195 for providing financial support.

\section{References}

Allen, C. W., 1972, Astrophysical Quantities (London: Athlone Press)

Chandrasekhar, S., 1956, ApJ, 124, 232

Donati, J.-F., \& Cameron, A. C., 1997, MNRAS, 291, 1

Hiremath, K. M \& Gokhale, M. H., 1996, Bull. Astron. Soc. India, 24, 345

Levy, E. H., in ASP Conf. Ser. Vol. 27, The Solar Cycle, ed. K. L. Harvey (San Francisco: ASP), 139 